\title{
A new two-level implicit scheme of order two in time and four in space based on half-step spline in compression approximations for unsteady 1D quasi-linear biharmonic equations
}

R.K. Mohanty ${ }^{1 *}$, Sachin Sharma ${ }^{2}$ and Swarn Singh ${ }^{3}$

${ }^{*}$ Correspondence:

rmohanty@sau.ac.in

1 Department of Applied

Mathematics, South Asian

University, New Delhi, India

Full list of author information is

available at the end of the article

\begin{abstract}
In this article, we discuss a new two-level implicit scheme of order of accuracy two in time and four in space based on the spline in compression approximations for the numerical solution of 1D unsteady quasi-linear biharmonic equations. We use only two half-step points and a central point on a uniform mesh for spline approximations and derivation of the method. The proposed method is derived directly from the continuity condition of the first order derivative of the spline function. For model linear problem, the proposed scheme is shown to be unconditionally stable. The proposed method has successfully tested on the Kuramoto-Sivashinsky equation and extended the Fisher-Kolmogorov equation. From the computational experiment, we obtain better numerical results compared to the results obtained by other researchers.
\end{abstract}

MSC: 65M06; 65M12;65M22;65Y20

Keywords: Quasi-linear biharmonic equations; Spline in compression function; Kuramoto-Sivashinsky equation; Newton's iterative method

\section{Introduction}

We consider the fourth order unsteady biharmonic equation with variable coefficient of the form

$$
A(x, t) u_{x x x x}+u_{t}=f\left(x, t, u, u_{x}, u_{x x}, u_{x x x}\right), \quad(x, t) \in \Omega,
$$

where $\Omega \equiv\{(x, t) \mid 0<x<1, t>0\}$ is the solution space.

The equation above may be written in a coupled manner as follows:

$$
\begin{aligned}
& u_{x x}=v, \quad(x, t) \in \Omega, \\
& A(x, t) v_{x x}=-u_{t}+f\left(x, t, u, u_{x}, v, v_{x}\right) \equiv g\left(x, t, u, v, u_{x}, v_{x}, u_{t}\right), \quad(x, t) \in \Omega .
\end{aligned}
$$

(c) The Author(s) 2018. This article is distributed under the terms of the Creative Commons Attribution 4.0 International License (http://creativecommons.org/licenses/by/4.0/), which permits unrestricted use, distribution, and reproduction in any medium, provided you give appropriate credit to the original author(s) and the source, provide a link to the Creative Commons license, and indicate if changes were made. 
The initial and boundary values are given by

$$
\begin{aligned}
& u(x, 0)=u_{0}(x), \quad 0 \leq x \leq 1, \\
& u(0, t)=a_{0}(t), \quad u(1, t)=a_{1}(t), \quad t>0, \\
& u_{x x}(0, t)=v(0, t)=b_{0}(t), \quad u_{x x}(1, t)=v(1, t)=b_{1}(t), \quad t>0,
\end{aligned}
$$

where $u_{0}, a_{0}, b_{0}, a_{1}$, and $b_{1}$ are smooth functions, and we assume that their required higher order derivatives exist in the solution region $\Omega$.

Many physical problems in terms of linear or nonlinear biharmonic equations are of common occurrence in engineering and science. Famous nonlinear PDEs of type (1.1) are generalized Kuramoto-Sivashinsky (GKS) equation, extended Fisher-Kolmogorov (EFK) equation, etc. The physical appearance and behavior of these equations were discussed in [1-10]. During the last decade, several numerical methods have been discussed for the solution of GKS and EFK equations. Xu and Shu [11] proposed a local discontinuous Galerkin method for the Kuramoto-Sivashinsky (KS) equation. Khater and Temsah [12] used a Chebyshev spectral collocation method to solve the GKS equation. Lai and Ma [13] studied a lattice Boltzmann method, and Uddin et al. [14] discussed a mesh-free method for the numerical solution of GKS equation. Using a B-spline collocation method, Mittal and Arora [15] and Lakestani and Dehghan [16] solved the GKS equation. Using a cubic Hermite collocation method, Ganaiea et al. [17] obtained the numerical solution of KS equation. Most recently, Mohanty and Kaur [18] developed a Numerov type compact variable mesh method for the solution of KS equation. A polynomial scaling function technique was used by Rashidinia and Jokar [19] for solving the GKS equation. Danumjaya and Pani [20] constructed a numerical scheme based on an orthogonal cubic spline collocation method for the solution of EFK equation. Using a splitting technique, Doss and Nandini [21] proposed an $H^{1}$-Galerkin mixed finite element method for the solution of EFK equation.

It is quite challenging to obtain the numerical solution of $1 \mathrm{D}$ unsteady nonlinear biharmonic equations due to their highly complex mechanism of solitary wave interaction. Over the last few decades, there has been a good amount of research work carried out for the numerical solution of biharmonic equation. In 1984, Stephenson [22] derived single cell discretizations of order two and four for the solution of biharmonic problems of first and second kind. Using three uniform grid points, a two-level implicit method of order two in time and four in space for the solution of (1.1) was constructed by Mohanty [23]. Most recently, Mohanty and Kaur [18] proposed a class of two-level implicit finite difference methods on a variable grid. Spline techniques are widely used for second order parabolic and hyperbolic PDEs in the literature. Jain et al. [24] discussed a finite difference method based on the spline in compression technique for the numerical solution of conservations laws. Kadalbajoo and Patidar [25] proposed a variable mesh spline in compression method for singular perturbation problems. Using second order consistency condition, Mohanty et al. $[26,27]$ presented a spline in compression method for hyperbolic and parabolic PDEs. Recently, Mohanty and Sharma [28, 29] derived new algorithms based on spline in compression approximations for the system of quasi-linear parabolic PDEs. To the authors' knowledge, no spline methods of order of accuracy two in time and four in space for the 
solution of nonlinear time-dependent biharmonic equation have been developed so far. In the present article, we propose a new two-level implicit numerical method of order of accuracy two in time and four in space, based on trigonometric spline approximations for the solution of PDE (1.1).

We use only two half-step points and one central point in $x$-direction at each time level, and no fictitious points are required for incorporating the boundary conditions. It is known that the difference methods for the biharmonic equation are based on five or more grid points in $x$-direction, and thus require fictitious points outside the solution region. These fictitious points are then eliminated by discretizing the given derivative boundary conditions. However, using the standard second order central differences for the boundary conditions, the accuracy of the overall numerical scheme is affected even if a higher order scheme is used at internal grid points. The algorithm presented in this paper reduces the fourth order PDE into coupled elliptic-parabolic equations, and we do not require to discretize the derivative boundary conditions. It attains order of accuracy two in time and four in space by using only three spatial grid points at each time level. The main attraction of this work is the application of the proposed high accuracy numerical method to the KS equation, GKS equation, and EFK equation.

The rest of this paper is organized as follows: In Sect. 2, we discuss the spline in compression function and its properties for the coupled equation. In Sect. 3, we present a new two-level implicit spline in compression method for 1D unsteady quasi-linear biharmonic problem of second kind, which is further derived in Sect. 4. In Sect. 5, the proposed spline in compression method is shown to be unconditionally stable for a linear biharmonic problem. In Sect. 6, we implement the proposed method on the KS equation, GKS equation, and EFK equation. We also compare our numerical results with the results of other researchers available in the literature. It is shown that the proposed numerical method yields better results as compared to the results given by other researchers. Concluding remarks are given in Sect. 7.

\section{Spline in compression approximations and their properties}

For the approximate solution of the proposed initial-boundary value problem, we discretize the space interval $[0,1]$ as $0=x_{0}<x_{1}<\cdots<x_{L}<x_{L+1}=1$, where $L$ is a positive integer. The proposed spline approximation consists of two half-step points $x_{l \pm 1 / 2}$ and a central point $x_{l}, l=0,1,2, \ldots, L$ with two end points $x_{0}$ and $x_{L+1}$. The neighboring halfstep points are defined as $x_{l-1 / 2}=x_{l}-\frac{h}{2}$ and $x_{l+1 / 2}=x_{l}+\frac{h}{2}, l=1(1) L$, where $h=x_{l+1}-x_{l}$, $l=0(1) L$ is the mesh size in $x$-direction and $k=t_{j+1}-t_{j}>0, j=0,1,2, \ldots$, is the mesh spacing in $t$-direction. Let $U_{l}^{j}=u\left(x_{l}, t_{j}\right)$ be the exact solution value of $u(x, t)$ approximated by $u_{l}^{j}$. Also let $V_{l}^{j}=v\left(x_{l}, t_{j}\right)$ be the exact solution value of $v(x, t)$ approximated by $v_{l}^{j}$. Now suppose $M=U_{x x}$ and $N=V_{x x}$.

A non-polynomial spline function which interpolates the value $U_{l}^{j}$ at $j$ th time level is given by

$$
\begin{aligned}
P_{j}(x)= & a_{l}^{j}+b_{l}^{j}\left(x-x_{l}\right)+c_{l}^{j} \sin \left[\tau\left(x-x_{l}\right)\right]+d_{l}^{j} \cos \left[\tau\left(x-x_{l}\right)\right], \\
& x_{l-1} \leq x \leq x_{l}, l=1(1) L+1, j>0,
\end{aligned}
$$

which satisfies the following properties at $j$ th time level:

(i) $P_{j}(x) \in C^{2}[0,1]$, 
(ii) $P_{j}\left(x_{l}\right)=U_{l}^{j}, P_{j}\left(x_{l-1}\right)=U_{l-1}^{j}$,

where $\tau$ is an arbitrary parameter and $P_{j}^{\prime \prime}\left(x_{l}\right)=M_{l}^{j}, P_{j}^{\prime \prime}\left(x_{l-1}\right)=M_{l-1}^{j}, P_{j}^{\prime \prime}\left(x_{l-1 / 2}\right)=M_{l-1 / 2}^{j}$.

Using these properties, we get the coefficients

$$
\begin{aligned}
& a_{l}^{j}=U_{l}^{j}+\frac{M_{l}^{j}}{\tau^{2}}, \quad b_{l}^{j}=\frac{U_{l}^{j}-U_{l-1}^{j}}{h}+\frac{M_{l}^{j}}{\tau \mu}-\frac{M_{l-1 / 2}^{j}}{\tau \mu} \cos \mu, \\
& c_{l}^{j}=\frac{M_{l-1 / 2}^{j}-M_{l}^{j} \cos \mu}{\tau^{2} \sin \mu}, \quad d_{l}^{j}=\frac{-M_{l}^{j}}{\tau^{2}},
\end{aligned}
$$

where $\mu=(\tau h / 2)$. Substituting the coefficients $a_{l}^{j}, b_{l}^{j}, c_{l}^{j}, d_{l}^{j}$ into Eq. (2.1), we obtain the spline in compression function $P_{j}(x)$ defined as

$$
\begin{aligned}
P_{j}(x)= & \left(U_{l}^{j}+\frac{M_{l}^{j}}{\tau^{2}}\right)+\left(\frac{U_{l}^{j}-U_{l-1}^{j}}{h}+\frac{M_{l}^{j}}{\tau \mu}-\frac{M_{l-1 / 2}^{j}}{\tau \mu} \cos \mu\right)\left(x-x_{l}\right) \\
& +\left(\frac{M_{l-1 / 2}^{j}-M_{l}^{j} \cos \mu}{\tau^{2} \sin \mu}\right) \sin \left[\tau\left(x-x_{l}\right)\right]-\frac{M_{l}^{j}}{\tau^{2}} \cos \left[\tau\left(x-x_{l}\right)\right], \\
& x \in\left[x_{l-1}, x_{l}\right], \\
P_{j}(x)= & \left(U_{l}^{j}+\frac{M_{l}^{j}}{\tau^{2}}\right)+\left(\frac{U_{l+1}^{j}-U_{l}^{j}}{h}-\frac{M_{l}^{j}}{\tau \mu}+\frac{M_{l+1 / 2}^{j}}{\tau \mu} \cos \mu\right)\left(x-x_{l}\right) \\
& +\left(\frac{M_{l}^{j} \cos \mu-M_{l+1 / 2}^{j}}{\tau^{2} \sin \mu}\right) \sin \left[\tau\left(x-x_{l}\right)\right]-\frac{M_{l}^{j}}{\tau^{2}} \cos \left[\tau\left(x-x_{l}\right)\right], \\
& x \in\left[x_{l}, x_{l+1}\right] .
\end{aligned}
$$

Similarly, a non-polynomial spline function which interpolates the value $V_{l}^{j}$ at $j$ th time level is given by

$$
\begin{aligned}
Q_{j}(x)= & \left(V_{l}^{j}+\frac{N_{l}^{j}}{\tau^{2}}\right)+\left(\frac{V_{l}^{j}-V_{l-1}^{j}}{h}+\frac{N_{l}^{j}}{\tau \mu}-\frac{N_{l-1 / 2}^{j}}{\tau \mu} \cos \mu\right)\left(x-x_{l}\right) \\
& +\left(\frac{N_{l-1 / 2}^{j}-N_{l}^{j} \cos \mu}{\tau^{2} \sin \mu}\right) \sin \left[\tau\left(x-x_{l}\right)\right]-\frac{N_{l}^{j}}{\tau^{2}} \cos \left[\tau\left(x-x_{l}\right)\right], \\
& x \in\left[x_{l-1}, x_{l}\right], \\
Q_{j}(x)= & \left(V_{l}^{j}+\frac{N_{l}^{j}}{\tau^{2}}\right)+\left(\frac{V_{l+1}^{j}-V_{l}^{j}}{h}-\frac{N_{l}^{j}}{\tau \mu}+\frac{N_{l+1 / 2}^{j}}{\tau \mu} \cos \mu\right)\left(x-x_{l}\right) \\
& +\left(\frac{N_{l}^{j} \cos \mu-N_{l+1 / 2}^{j}}{\tau^{2} \sin \mu}\right) \sin \left[\tau\left(x-x_{l}\right)\right]-\frac{N_{l}^{j}}{\tau^{2}} \cos \left[\tau\left(x-x_{l}\right)\right], \\
& x \in\left[x_{l}, x_{l+1}\right],
\end{aligned}
$$

where $Q_{j}(x)$ satisfies the following properties at $j$ th time level:

(i) $Q_{j}(x) \in C^{2}[0,1]$,

(ii) $Q_{j}\left(x_{l}\right)=V_{l}^{j}, Q_{j}\left(x_{l-1}\right)=V_{l-1}^{j}$, and $Q_{j}^{\prime \prime}\left(x_{l}\right)=N_{l}^{j}, Q_{j}^{\prime \prime}\left(x_{l-1}\right)=N_{l-1}^{j}, Q_{j}^{\prime \prime}\left(x_{l-1 / 2}\right)=N_{l-1 / 2}^{j}$. 
Using the continuity of the first derivative of $P_{j}(x)$ and $Q_{j}(x)$, that is, $P_{j}^{\prime}\left(x_{l}-\right)=P_{j}^{\prime}\left(x_{l}+\right)$ and $Q_{j}^{\prime}\left(x_{l}-\right)=Q_{j}^{\prime}\left(x_{l}+\right)$, we obtain the following consistency conditions:

$$
\begin{aligned}
& \frac{U_{l+1}^{j}-2 U_{l}^{j}+U_{l-1}^{j}}{h^{2}}=\alpha M_{l+1 / 2}^{j}+2 \beta M_{l}^{j}+\alpha M_{l-1 / 2}^{j}+O\left(h^{4}\right), \quad l=1(1) L, \\
& \frac{V_{l+1}^{j}-2 V_{l}^{j}+V_{l-1}^{j}}{h^{2}}=\alpha N_{l+1 / 2}^{j}+2 \beta N_{l}^{j}+\alpha N_{l-1 / 2}^{j}+O\left(h^{4}\right), \quad l=1(1) L,
\end{aligned}
$$

where

$$
\begin{aligned}
& \alpha=\frac{1}{2 \mu^{2}}\left[\frac{\mu}{\sin \mu}-\cos \mu\right]=\frac{1}{3}-\frac{\mu^{2}}{90}+O\left(\mu^{4}\right), \\
& \beta=\frac{1}{2 \mu^{2}}(1-\mu \cot \mu)=\frac{1}{6}+\frac{\mu^{2}}{90}+O\left(\mu^{4}\right) .
\end{aligned}
$$

On equating the coefficients of $M_{l}^{j}$ and $N_{l}^{j}$ in (2.7)-(2.8), we obtain the condition

$$
\alpha+\beta=1 / 2 \text {. }
$$

Substituting the values of $\alpha$ and $\beta$ in (2.10) and neglecting $O\left(\mu^{4}\right)$ terms, we get

$$
\tan (\mu / 2)=\mu / 2
$$

The above equation has an infinite number of roots, the smallest positive non-zero root being given by $\mu=8.986818916$.

Further, from (2.2)-(2.5), we get

$$
\begin{aligned}
& P_{j}^{\prime}\left(x_{l-1 / 2}\right)=\frac{U_{l}^{j}-U_{l-1}^{j}}{h}-\frac{h}{4}\left(2 \beta M_{l}^{j}-\alpha M_{l-1 / 2}^{j}\right), \\
& P_{j}^{\prime}\left(x_{l+1 / 2}\right)=\frac{U_{l+1}^{j}-U_{l}^{j}}{h}+\frac{h}{4}\left(2 \beta M_{l}^{j}-\alpha M_{l+1 / 2}^{j}\right), \\
& Q_{j}^{\prime}\left(x_{l-1 / 2}\right)=\frac{V_{l}^{j}-V_{l-1}^{j}}{h}-\frac{h}{4}\left(2 \beta N_{l}^{j}-\alpha N_{l-1 / 2}^{j}\right), \\
& Q_{j}^{\prime}\left(x_{l+1 / 2}\right)=\frac{V_{l+1}^{j}-V_{l}^{j}}{h}+\frac{h}{4}\left(2 \beta N_{l}^{j}-\alpha N_{l+1 / 2}^{j}\right) .
\end{aligned}
$$

Relations (2.12)-(2.15) are important properties of spline functions.

\section{Formulation of a spline in compression method}

In order to derive the high accuracy numerical method based on a spline function and its properties for PDEs (1.2)-(1.3), we require the following three point approximations:

For $r=0, \pm 1$, we denote

$$
\begin{aligned}
& \bar{t}_{j}=t_{j}+\theta k, \\
& \bar{A}_{l}^{j}=A\left(x_{l}, \bar{t}_{j}\right), \\
& \bar{A}_{x l}^{j}=A_{x}\left(x_{l}, \bar{t}_{j}\right),
\end{aligned}
$$




$$
\begin{aligned}
& \bar{A}_{x x l}^{j}=A_{x x}\left(x_{l}, \bar{t}_{j}\right), \\
& \bar{U}_{l \pm r}^{j}=\theta U_{l \pm r}^{j+1}+(1-\theta) U_{l \pm r}^{j}, \\
& \bar{U}_{l \pm 1 / 2}^{j}=\frac{1}{2}\left(\bar{U}_{l \pm 1}^{j}+\bar{U}_{l}^{j}\right), \\
& \bar{V}_{l \pm r}^{j}=\theta V_{l \pm r}^{j+1}+(1-\theta) V_{l \pm r}^{j}, \\
& \bar{V}_{l \pm 1 / 2}^{j}=\frac{1}{2}\left(\bar{V}_{l \pm 1}^{j}+\bar{V}_{l}^{j}\right) \\
& \bar{U}_{t l \pm r}^{j}=\frac{1}{k}\left(U_{l \pm r}^{j+1}-U_{l \pm r}^{j}\right), \\
& \bar{U}_{t l \pm 1 / 2}^{j}=\frac{1}{2 k}\left(U_{l \pm 1}^{j+1}+U_{l}^{j+1}-U_{l \pm 1}^{j}-U_{l}^{j}\right), \\
& \bar{U}_{x l}^{j}=\frac{\bar{U}_{l+1}^{j}-\bar{U}_{l-1}^{j}}{2 h}, \\
& \bar{U}_{x l+1 / 2}^{j}=\frac{\bar{U}_{l+1}^{j}-\bar{U}_{l}^{j}}{h}, \\
& \bar{U}_{x l-1 / 2}^{j}=\frac{\bar{U}_{l}^{j}-\bar{U}_{l-1}^{j}}{h}, \\
& \bar{V}_{x l}^{j}=\frac{\bar{V}_{l+1}^{j}-\bar{V}_{l-1}^{j}}{2 h}, \\
& \bar{V}_{x l+1 / 2}^{j}=\frac{\bar{V}_{l+1}^{j}-\bar{V}_{l}^{j}}{h}, \\
& \bar{V}_{x l-1 / 2}^{j}=\frac{\bar{V}_{l}^{j}-\bar{V}_{l-1}^{j}}{h}, \\
& \bar{U}_{x x l}^{j}=\frac{\bar{U}_{l+1}^{j}-2 \bar{U}_{l}^{j}+\bar{U}_{l-1}^{j}}{h^{2}}, \\
& \bar{V}_{x x l}^{j}=\frac{\bar{V}_{l+1}^{j}-2 \bar{V}_{l}^{j}+\bar{V}_{l-1}^{j}}{h^{2}},
\end{aligned}
$$

where $\theta=\frac{1}{2}$.

Also define

$$
M_{l}^{j}=V_{l}^{j}, \quad M_{l \pm 1 / 2}^{j}=V_{l \pm 1 / 2}^{j},
$$

and

$$
N_{l}^{j}=\frac{1}{A_{l}^{j}}\left[-U_{t l}^{j}+F_{l}^{j}\right], \quad N_{l \pm 1 / 2}^{j}=\frac{1}{A_{l \pm 1 / 2}^{j}}\left[-U_{t l \pm 1 / 2}^{j}+F_{l \pm 1 / 2}^{j}\right] .
$$

Further, we define the following approximations:

$$
\begin{aligned}
& \bar{F}_{l}^{j}=f\left(x_{l}, \bar{t}_{j}, \bar{U}_{l}^{j}, \bar{V}_{l}^{j}, \bar{U}_{x l}^{j}, \bar{V}_{x l}^{j}\right), \\
& \bar{F}_{l+1 / 2}^{j}=f\left(x_{l+1 / 2}, \bar{t}_{j}, \bar{U}_{l+1 / 2}^{j}, \bar{V}_{l+1 / 2}^{j}, \bar{U}_{x l+1 / 2}^{j}, \bar{V}_{x l+1 / 2}^{j}\right), \\
& \bar{F}_{l-1 / 2}^{j}=f\left(x_{l-1 / 2}, \bar{t}_{j}, \bar{U}_{l-1 / 2}^{j}, \bar{V}_{l-1 / 2}^{j}, \bar{U}_{x l-1 / 2}^{j}, \bar{V}_{x l-1 / 2}^{j}\right), \\
& \bar{M}_{l}^{j}=\bar{V}_{l}^{j},
\end{aligned}
$$




$$
\begin{aligned}
& \bar{M}_{l+1 / 2}^{j}=\bar{V}_{l+1 / 2}^{j}, \\
& \bar{M}_{l-1 / 2}^{j}=\bar{V}_{l-1 / 2}^{j}, \\
& \bar{N}_{l}^{j}=\frac{1}{\bar{A}_{l}^{j}}\left[-\bar{U}_{t l}^{j}+\bar{F}_{l}^{j}\right], \\
& \bar{N}_{l+1 / 2}^{j}=\frac{1}{\bar{A}_{l+1 / 2}^{j}}\left[-\bar{U}_{t l+1 / 2}^{j}+\bar{F}_{l+1 / 2}^{j}\right], \\
& \bar{N}_{l-1 / 2}^{j}=\frac{1}{\bar{A}_{l-1 / 2}^{j}}\left[-\bar{U}_{t l-1 / 2}^{j}+\bar{F}_{l-1 / 2}^{j}\right] .
\end{aligned}
$$

From the properties of spline functions (2.12)-(2.15), we have the following approximations:

$$
\begin{aligned}
& \hat{U}_{x l-1 / 2}^{j}=\frac{\bar{U}_{l}^{j}-\bar{U}_{l-1}^{j}}{h}-\frac{h}{4}\left(2 \beta \bar{M}_{l}^{j}-\alpha \bar{M}_{l-1 / 2}^{j}\right), \\
& \hat{U}_{x l+1 / 2}^{j}=\frac{\bar{U}_{l+1}^{j}-\bar{U}_{l}^{j}}{h}+\frac{h}{4}\left(2 \beta \bar{M}_{l}^{j}-\alpha \bar{M}_{l+1 / 2}^{j}\right), \\
& \hat{V}_{x l-1 / 2}^{j}=\frac{\bar{V}_{l}^{j}-\bar{V}_{l-1}^{j}}{h}-\frac{h}{4}\left(2 \beta \bar{N}_{l}^{j}-\alpha \bar{N}_{l-1 / 2}^{j}\right), \\
& \hat{V}_{x l+1 / 2}^{j}=\frac{\bar{V}_{l+1}^{j}-\bar{V}_{l}^{j}}{h}+\frac{h}{4}\left(2 \beta \bar{N}_{l}^{j}-\alpha \bar{N}_{l+1 / 2}^{j}\right) .
\end{aligned}
$$

We consider the following linear combinations in order to increase the accuracy of the scheme:

$$
\begin{aligned}
& \hat{U}_{l}^{j}=\bar{U}_{l}^{j}+a h^{2} \bar{U}_{x x l}^{j}, \\
& \hat{V}_{l}^{j}=\bar{V}_{l}^{j}+b h^{2} \bar{V}_{x x l}^{j}, \\
& \hat{U}_{x l}^{j}=\bar{U}_{x l}^{j}+\operatorname{ch}\left(\bar{M}_{l+1 / 2}^{j}-\bar{M}_{l-1 / 2}^{j}\right), \\
& \hat{V}_{x l}^{j}=\bar{V}_{x l}^{j}+\operatorname{dh}\left(\bar{N}_{l+1 / 2}^{j}-\bar{N}_{l-1 / 2}^{j}\right),
\end{aligned}
$$

where $a=b=\frac{-1}{4}$ and $c=d=\frac{-1}{6}$.

Further, we define

$$
\begin{aligned}
& \hat{F}_{l}^{j}=f\left(x_{l}, \bar{t}_{j}, \hat{U}_{l}^{j}, \hat{V}_{l}^{j}, \hat{U}_{x l}^{j}, \hat{V}_{x l}^{j}\right), \\
& \hat{F}_{l+1 / 2}^{j}=f\left(x_{l+1 / 2}, \bar{t}_{j}, \bar{U}_{l+1 / 2}^{j}, \bar{V}_{l+1 / 2}^{j}, \hat{U}_{x l+1 / 2}^{j}, \hat{V}_{x l+1 / 2}^{j}\right), \\
& \hat{F}_{l-1 / 2}^{j}=f\left(x_{l-1 / 2}, \bar{t}_{j}, \bar{U}_{l-1 / 2}^{j}, \bar{V}_{l-1 / 2}^{j}, \hat{U}_{x l-1 / 2}^{j}, \hat{V}_{x l-1 / 2}^{j}\right) .
\end{aligned}
$$

Then, at each grid point $\left(x_{l}, t_{j}\right)$, the spline in compression method for the system of differential equations (1.2)-(1.3) is given by

$$
\bar{U}_{l+1}^{j}-2 \bar{U}_{l}^{j}+\bar{U}_{l-1}^{j}=\frac{h^{2}}{3}\left(\bar{V}_{l+1 / 2}^{j}+\hat{V}_{l}^{j}+\bar{V}_{l-1 / 2}^{j}\right)+\hat{T}_{1 l}^{j},
$$




$$
\begin{aligned}
& {\left[\bar{A}_{l}^{j}+\frac{h^{2}}{12}\left(\bar{A}_{x x l}^{j}-\frac{2\left(\bar{A}_{x l}^{j}\right)^{2}}{\bar{A}_{l}^{j}}\right)\right]\left[\bar{V}_{l+1}^{j}-2 \bar{V}_{l}^{j}+\bar{V}_{l-1}^{j}\right]} \\
& \quad+\frac{h^{2}}{12}\left[10 \bar{U}_{t l}^{j}+\left(1-\frac{h \bar{A}_{x l}^{j}}{\bar{A}_{l}^{j}}\right) \bar{U}_{t l+1}^{j}+\left(1+\frac{h \bar{A}_{x l}^{j}}{\bar{A}_{l}^{j}}\right) \bar{U}_{t l-1}^{j}\right] \\
& =\frac{h^{2}}{3}\left[\left(1-\frac{h \bar{A}_{x l}^{j}}{2 \bar{A}_{l}^{j}}\right) \hat{F}_{l+1 / 2}^{j}+\left(1+\frac{h \bar{A}_{x l}^{j}}{2 \bar{A}_{l}^{j}}\right) \hat{F}_{l-1 / 2}^{j}+\hat{F}_{l}^{j}\right]+\hat{T}_{2 l}^{j},
\end{aligned}
$$

where truncation errors $\hat{T}_{1 l}^{j}=O\left(k^{2} h^{2}+h^{6}\right)$ and $\hat{T}_{2 l}^{j}=O\left(k^{2} h^{2}+k h^{4}+h^{6}\right)$.

\section{Derivation of the numerical algorithm}

In this section, we derive the spline method from the consistency conditions given by (2.6)-(2.7).

Substituting the values

$$
\begin{aligned}
& M_{l}^{j}=V_{l}^{j}, \quad M_{l \pm \frac{1}{2}}^{j}=V_{l \pm \frac{1}{2}}^{j}, \quad N_{l}^{j}=\frac{1}{A_{l}^{j}}\left[-U_{t l}^{j}+F_{l}^{j}\right], \\
& N_{l \pm 1 / 2}^{j}=\frac{1}{A_{l \pm 1 / 2}^{j}}\left[-U_{t l \pm 1 / 2}^{j}+F_{l \pm 1 / 2}^{j}\right] \quad \text { into (2.6)-(2.7), }
\end{aligned}
$$

we get

$$
\begin{aligned}
U_{l+1}^{j}-2 U_{l}^{j}+U_{l-1}^{j}= & \frac{h^{2}}{3}\left(V_{l+1 / 2}^{j}+V_{l}^{j}+V_{l-1 / 2}^{j}\right)+O\left(h^{6}\right), \\
V_{l+1}^{j}-2 V_{l}^{j}+V_{l-1}^{j}= & \frac{h^{2}}{3}\left[\frac{1}{A_{l+1 / 2}^{j}}\left(-U_{t l+1 / 2}^{j}+F_{l+1 / 2}^{j}\right)+\frac{1}{A_{l-1 / 2}^{j}}\left(-U_{t l-1 / 2}^{j}+F_{l-1 / 2}^{j}\right)\right. \\
& \left.+\frac{1}{A_{l}^{j}}\left(-U_{t l}^{j}+F_{l}^{j}\right)\right]+O\left(h^{6}\right) .
\end{aligned}
$$

At the mesh point $\left(x_{l}, t_{j}\right)$, let us denote

$$
\begin{array}{lll}
\frac{\partial^{p+q} U_{l}^{j}}{\partial x^{p} \partial t^{q}}=U_{p q}, & \frac{\partial^{p+q} V_{l}^{j}}{\partial x^{p} \partial t^{q}}=V_{p q}, & \frac{\partial^{p+q} A_{l}^{j}}{\partial x^{p} \partial t^{q}}=A_{p q}, \quad \text { and } \\
\alpha_{l}^{(1) j}=\left(\frac{\partial f}{\partial U}\right)_{l}^{j}, & \alpha_{l}^{(2) j}=\left(\frac{\partial f}{\partial V}\right)_{l}^{j}, & \beta_{l}^{(1) j}=\left(\frac{\partial f}{\partial U_{x}}\right)_{l}^{j}, \quad \beta_{l}^{(2) j}=\left(\frac{\partial f}{\partial V_{x}}\right)_{l}^{j}, \\
\xi_{l}^{j}=\left(\frac{\partial f}{\partial t}\right)_{l}^{j} &
\end{array}
$$

To simplify (4.2), we need the following approximations:

$$
\begin{aligned}
& \frac{1}{A_{l \pm 1 / 2}^{j}}=\frac{1}{A_{00}}\left[1 \mp \frac{h A_{10}}{2 A_{00}}-\frac{h^{2}}{8 A_{00}}\left\{A_{20}-2 \frac{\left(A_{10}\right)^{2}}{A_{00}}\right\} \pm O\left(h^{3}\right)\right], \\
& U_{t l \pm 1 / 2}^{j}=U_{t l}^{j} \pm \frac{h}{2} U_{11}+\frac{h^{2}}{8} U_{21} \pm O\left(h^{3}\right), \\
& 2 h U_{11}=\left(U_{t l+1}^{j}-U_{t l-1}^{j}\right)+O\left(h^{3}\right), \\
& h^{2} U_{21}=\left(U_{t l+1}^{j}-2 U_{t l}^{j}+U_{t l-1}^{j}\right)+O\left(h^{4}\right),
\end{aligned}
$$




$$
\begin{aligned}
& F_{l+1 / 2}^{j}+F_{l-1 / 2}^{j}=2 F_{l}^{j}+O\left(h^{2}\right)=2\left(A_{00} V_{20}+U_{01}\right)+O\left(h^{2}\right), \\
& h^{2} V_{20}=V_{l+1}^{j}-2 V_{l}^{j}+V_{l-1}^{j}+O\left(h^{4}\right) .
\end{aligned}
$$

With the help of (4.3)-(4.8), the consistency conditions (4.1)-(4.2) may be re-written as follows:

$$
\begin{aligned}
& U_{l+1}^{j}-2 U_{l}^{j}+U_{l-1}^{j}=\frac{h^{2}}{3}\left(V_{l+1 / 2}^{j}+V_{l}^{j}+V_{l-1 / 2}^{j}\right)+O\left(h^{6}\right), \\
& {\left[A_{00}+\frac{h^{2}}{12}\left(A_{20}-\frac{2\left(A_{10}\right)^{2}}{A_{00}}\right)\right]\left[V_{l+1}^{j}-2 V_{l}^{j}+V_{l-1}^{j}\right]} \\
& \quad+\frac{h^{2}}{12}\left[10 U_{t l}^{j}+\left(1-\frac{h A_{10}}{A_{00}}\right) U_{t l+1}^{j}+\left(1+\frac{h A_{10}}{A_{00}}\right) U_{t l-1}^{j}\right] \\
& \quad=\frac{h^{2}}{3}\left[\left(1-\frac{h A_{10}}{2 A_{00}}\right) F_{l+1 / 2}^{j}+\left(1+\frac{h A_{10}}{2 A_{00}}\right) F_{l-1 / 2}^{j}+F_{l}^{j}\right]+O\left(h^{6}\right) .
\end{aligned}
$$

Simplifying approximations (3.5)-(3.18) using a Taylor series expansion, we obtain

$$
\begin{aligned}
& \bar{u}_{l}^{j}=U_{l}^{j}+\theta k U_{01}+O\left(k^{2}\right), \\
& \bar{U}_{l \pm 1}^{j}=U_{l \pm 1}^{j}+\theta k\left(U_{01} \pm h U_{11}\right)+O\left(k^{2}\right), \\
& \bar{U}_{l \pm 1 / 2}^{j}=U_{l \pm 1 / 2}^{j}+\theta k U_{01}+\frac{h^{2}}{8} U_{20}+O\left(k^{2}+k h^{2}+h^{4}\right), \\
& \bar{V}_{l}^{j}=V_{l}^{j}+\theta k V_{01}+O\left(k^{2}\right), \\
& \bar{V}_{l \pm 1}^{j}=V_{l \pm 1}^{j}+\theta k\left(V_{01} \pm h V_{11}\right)+O\left(k^{2}\right), \\
& \bar{V}_{l \pm 1 / 2}^{j}=V_{l \pm 1 / 2}^{j}+\theta k V_{01}+\frac{h^{2}}{8} V_{20}+O\left(k^{2}+k h^{2}+h^{4}\right), \\
& \bar{u}_{t l}^{j}=U_{01}+\frac{k}{2} U_{02}+O\left(k^{2}\right) \text {, } \\
& \bar{U}_{t l \pm 1}^{j}=U_{t l \pm 1}^{j}+\frac{k}{2} U_{02}+O\left(k h+k^{2}\right), \\
& \bar{U}_{t l \pm 1 / 2}^{j}=U_{t l \pm 1 / 2}^{j}+\frac{k}{2} U_{02}+\frac{h^{2}}{8} U_{21}+O\left(k^{2}+k h^{2}+h^{4}\right), \\
& \bar{U}_{x l}^{j}=U_{10}+\frac{h^{2}}{6} U_{30}+\theta k U_{11}+O\left(k^{2}+h^{4}\right), \\
& \bar{U}_{x l \pm 1 / 2}^{j}=U_{x l \pm 1 / 2}^{j}+\frac{h^{2}}{24} U_{30}+\theta k U_{11}+O\left(k^{2}+k h^{2}+h^{4}\right), \\
& \bar{V}_{x l}^{j}=V_{10}+\frac{h^{2}}{6} V_{30}+\theta k V_{11}+O\left(k^{2}+h^{4}\right), \\
& \bar{V}_{x l \pm 1 / 2}^{j}=V_{x l \pm 1 / 2}^{j}+\frac{h^{2}}{24} V_{30}+\theta k V_{11}+O\left(k^{2}+k h^{2}+h^{4}\right), \\
& \bar{U}_{l+1}^{j}-2 \bar{U}_{l}^{j}+\bar{U}_{l-1}^{j}=\left(U_{l+1}^{j}-2 U_{l}^{j}+U_{l-1}^{j}\right)+\theta k U_{21}+O\left(k^{2} h^{2}+k h^{4}\right) \text {, } \\
& \bar{V}_{l+1}^{j}-2 \bar{V}_{l}^{j}+\bar{V}_{l-1}^{j}=\left(V_{l+1}^{j}-2 V_{l}^{j}+V_{l-1}^{j}\right)+\theta k V_{21}+O\left(k^{2} h^{2}+k h^{4}\right) \text {, } \\
& \bar{U}_{x x l}^{j}=U_{x x l}^{j}+\theta k U_{21}+\frac{h^{2}}{12} U_{40}+O\left(k^{2}+k h^{2}+h^{4}\right) \text {, } \\
& \bar{V}_{x x l}^{j}=V_{x x l}^{j}+\theta k V_{21}+\frac{h^{2}}{12} V_{40}+O\left(k^{2}+k h^{2}+h^{4}\right) \text {. }
\end{aligned}
$$


With the help of (4.11)-(4.23), from (3.19)-(3.21), we obtain

$$
\begin{aligned}
\bar{F}_{l}^{j}=F_{l}^{j}+ & \theta k\left[\xi_{l}^{j}+U_{01} \alpha_{l}^{(1) j}+V_{01} \alpha_{l}^{(2) j}+U_{11} \beta_{l}^{(1) j}+V_{11} \beta_{l}^{(2) j}\right] \\
+ & \frac{h^{2}}{6}\left[U_{30} \beta_{l}^{(1) j}+V_{30} \beta_{l}^{(2) j}\right]+O\left(k^{2}+h^{4}\right), \\
\bar{F}_{l \pm 1 / 2}^{j}= & F_{l \pm 1 / 2}^{j}+\theta k\left[\xi_{l}^{j}+U_{01} \alpha_{l}^{(1) j}+V_{01} \alpha_{l}^{(2) j}+U_{11} \beta_{l}^{(1) j}+V_{11} \beta_{l}^{(2) j}\right] \\
& +\frac{h^{2}}{24}\left[3 U_{20} \alpha_{l}^{(1) j}+3 V_{20} \alpha_{l}^{(2) j}+U_{30} \beta_{l}^{(1) j}+V_{30} \beta_{l}^{(2) j}\right] \\
& +O\left( \pm k h \pm h^{3}+k^{2}+k h^{2}+h^{4}\right) .
\end{aligned}
$$

Further, we can write

$$
\begin{aligned}
& \frac{1}{\bar{A}_{l}^{j}}=\frac{1}{A\left(x_{l}, \bar{t}_{j}\right)}=\frac{1}{A\left(x_{l}, t_{j}+\theta k\right)}=\frac{1}{A_{l}^{j}}\left[1-\theta k \frac{A_{t l}^{j}}{A_{l}^{j}}+O\left(k^{2}\right)\right], \\
& \frac{1}{\bar{A}_{l \pm 1 / 2}^{j}}=\frac{1}{A_{l \pm 1 / 2}^{j}}\left[1-\theta k \frac{A_{t l \pm 1 / 2}^{j}}{A_{l \pm 1 / 2}^{j}}+O\left(k^{2}\right)\right] .
\end{aligned}
$$

Using (4.28)-(4.31) and simplifying (3.22)-(3.27), we obtain

$$
\begin{aligned}
& \bar{M}_{l}^{j}=U_{x x l}^{j}+O\left(k+h^{2}\right), \\
& \bar{M}_{l \pm 1 / 2}^{j}=U_{x x l \pm 1 / 2}^{j}+O\left(k+h^{2}\right), \\
& \bar{N}_{l}^{j}=V_{x x l}^{j}+O\left(k+h^{2}\right), \\
& \bar{N}_{l \pm 1 / 2}^{j}=V_{x x l \pm 1 / 2}^{j}+O\left(k+h^{2}\right) .
\end{aligned}
$$

Using (4.32)-(4.35) in (3.28)-(3.31), we obtain

$$
\begin{aligned}
& \hat{U}_{x l \pm 1 / 2}^{j}=U_{x l \pm 1 / 2}^{j}+\theta k U_{11}+O\left( \pm k h \pm h^{3}+k^{2}+k h^{2}+h^{4}\right), \\
& \hat{V}_{x l \pm 1 / 2}^{j}=V_{x l \pm 1 / 2}^{j}+\theta k V_{11}+O\left( \pm k h \pm h^{3}+k^{2}+k h^{2}+h^{4}\right) .
\end{aligned}
$$

Simplifying (3.37)-(3.38), we obtain

$$
\begin{aligned}
\hat{F}_{l \pm 1 / 2}^{j}= & F_{l \pm 1 / 2}^{j}+\theta k\left[\xi_{l}^{j}+U_{01} \alpha_{l}^{(1) j}+V_{01} \alpha_{l}^{(2) j}+U_{11} \beta_{l}^{(1) j}+V_{11} \beta_{l}^{(2) j}\right] \\
& +\frac{h^{2}}{8}\left[U_{20} \alpha_{l}^{(1) j}+V_{20} \alpha_{l}^{(2) j}\right]+O\left( \pm k h \pm h^{3}+k^{2}+k h^{2}+h^{4}\right) .
\end{aligned}
$$

Now, using the above approximations and simplifying (3.32)-(3.35), we obtain

$$
\begin{aligned}
& \hat{U}_{l}^{j}=U_{l}^{j}+\theta k U_{01}+a h^{2} U_{20}+O\left(k^{2}+k h^{2}+h^{4}\right), \\
& \hat{V}_{l}^{j}=V_{l}^{j}+\theta k V_{01}+b h^{2} V_{20}+O\left(k^{2}+k h^{2}+h^{4}\right), \\
& \hat{U}_{x l}^{j}=U_{x l}^{j}+\theta k U_{11}+\frac{h^{2}}{6}[1+6 c] U_{30}+O\left(k^{2}+k h^{2}+h^{4}\right), \\
& \hat{V}_{x l}^{j}=V_{x l}^{j}+\theta k V_{11}+\frac{h^{2}}{6}[1+6 d] V_{30}+O\left(k^{2}+k h^{2}+h^{4}\right) .
\end{aligned}
$$


Equating the coefficient of $h^{2}$ to zero in Eqs. (4.41) and (4.42), we obtain $c=d=\frac{-1}{6}$, and Eqs. (4.41) and (4.42) reduce to

$$
\begin{aligned}
& \hat{U}_{x l}^{j}=U_{x l}^{j}+\theta k U_{11}+O\left(k^{2}+k h^{2}+h^{4}\right), \\
& \hat{V}_{x l}^{j}=V_{x l}^{j}+\theta k V_{11}+O\left(k^{2}+k h^{2}+h^{4}\right) .
\end{aligned}
$$

With the help of (4.39)-(4.44), from (3.36), we obtain

$$
\begin{aligned}
\hat{F}_{l}^{j}= & F_{l}^{j}+\theta k\left[\xi_{l}^{j}+U_{01} \alpha_{l}^{(1) j}+V_{01} \alpha_{l}^{(2) j}+U_{11} \beta_{l}^{(1) j}+V_{11} \beta_{l}^{(2) j}\right] \\
& +h^{2}\left[a U_{20} \beta_{l}^{(1) j}+b V_{20} \beta_{l}^{(2) j}\right]+O\left(k^{2}+k h^{2}+h^{4}\right) .
\end{aligned}
$$

At the grid point $\left(x_{l}, t_{j}\right)$, differentiating (1.2)-(1.3) with respect to ' $t$ ', we obtain a relation

$$
\begin{aligned}
& U_{21}=V_{01}, \\
& A_{00} V_{21}+A_{01} V_{20}=-U_{02}+\xi_{l}^{j}+U_{01} \alpha_{l}^{(1) j}+V_{01} \alpha_{l}^{(2) j}+U_{11} \beta_{l}^{(1) j}+V_{11} \beta_{l}^{(2) j} .
\end{aligned}
$$

Using (4.46)-(4.47), we may re-write (4.38) and (4.45) as

$$
\begin{aligned}
\hat{F}_{l \pm 1 / 2}^{j}= & F_{l \pm 1 / 2}^{j}+\theta k\left[A_{00} V_{21}+A_{01} V_{20}+U_{02}\right]+\frac{h^{2}}{8}\left[U_{20} \alpha_{l}^{(1) j}+V_{20} \alpha_{l}^{(2) j}\right], \\
& +O\left( \pm k h \pm h^{3}+k^{2}+k h^{2}+h^{4}\right), \\
\hat{F}_{l}^{j}= & F_{l}^{j}+\theta k\left[A_{00} V_{21}+A_{01} V_{20}+U_{02}\right]+\frac{h^{2}}{8}\left[a U_{20} \alpha_{l}^{(1) j}+b V_{20} \alpha_{l}^{(2) j}\right] \\
+ & O\left(k^{2}+k h^{2}+h^{4}\right) .
\end{aligned}
$$

It is easy to verify that

$$
\begin{aligned}
& \bar{A}_{l}^{j}=A_{00}+\theta k A_{01}+O\left(k^{2}\right), \\
& \bar{A}_{x l}^{j}=A_{10}+\theta k A_{11}+O\left(k^{2}\right) \text {, } \\
& \bar{A}_{x x l}^{j}=A_{20}+\theta k A_{21}+O\left(k^{2}\right) \text {. } \\
& \bar{U}_{l+1}^{j}-2 \bar{U}_{l}^{j}+\bar{U}_{l-1}^{j}=U_{l+1}^{j}-2 U_{l}^{j}+U_{l-1}^{j}+\theta k h^{2} U_{21}+O\left(k^{2} h^{2}+k h^{4}\right), \\
& \bar{V}_{l+1}^{j}-2 \bar{V}_{l}^{j}+\bar{V}_{l-1}^{j}=V_{l+1}^{j}-2 V_{l}^{j}+V_{l-1}^{j}+\theta k h^{2} V_{21}+O\left(k^{2} h^{2}+k h^{4}\right), \\
& {\left[\bar{A}_{l}^{j}+\frac{h^{2}}{12}\left(\bar{A}_{x x l}^{j}-\frac{2\left(\bar{A}_{x l}^{j}\right)^{2}}{\bar{A}_{l}^{j}}\right)\right]\left[\bar{V}_{l+1}^{j}-2 \bar{V}_{l}^{j}+\bar{V}_{l-1}^{j}\right]} \\
& =\left[A_{l}^{j}+\frac{h^{2}}{12}\left(A_{x x l}^{j}-\frac{2\left(A_{x l}^{j}\right)^{2}}{A_{l}^{j}}\right)\right]\left[V_{l+1}^{j}-2 V_{l}^{j}+V_{l-1}^{j}\right]+\theta k h^{2}\left[A_{00} V_{21}+A_{01} V_{20}\right] \\
& +O\left(k^{2} h^{2}+k h^{4}+h^{6}\right) \\
& \left(1-\frac{h \bar{A}_{x l}^{j}}{\bar{A}_{l}^{j}}\right) \bar{U}_{t l+1}^{j}=\left(1-\frac{h A_{10}}{A_{00}}\right) U_{t l+1}^{j}+\frac{k}{2} U_{20}+O\left(k h+k^{2}+k h^{2}\right), \\
& \left(1+\frac{h \bar{A}_{x l}^{j}}{\bar{A}_{l}^{j}}\right) \bar{U}_{t l-1}^{j}=\left(1+\frac{h A_{10}}{A_{00}}\right) U_{t l-1}^{j}+\frac{k}{2} U_{20}+O\left(-k h+k^{2}+k h^{2}\right),
\end{aligned}
$$




$$
\begin{aligned}
& 10 \bar{U}_{t l}^{j}+\left(1-\frac{h \bar{A}_{x l}^{j}}{\bar{A}_{l}^{j}}\right) \bar{U}_{t l+1}^{j}+\left(1+\frac{h \bar{A}_{x l}^{j}}{\bar{A}_{l}^{j}}\right) \bar{U}_{t l-1}^{j} \\
& =10 U_{t l}^{j}+\left(1-\frac{h A_{x l}^{j}}{A_{l}^{j}}\right) U_{t l+1}^{j}+\left(1+\frac{h A_{x l}^{j}}{A_{l}^{j}}\right) U_{t l-1}^{j}+6 k U_{02} \\
& \quad+O\left(k^{2}+k h^{2}+h^{4}\right) .
\end{aligned}
$$

With the help of approximations (4.48)-(4.58), from (3.39)-(3.40), we obtain

$$
\begin{aligned}
& \left(U_{l+1}^{j}-2 U_{l}^{j}+U_{l-1}^{j}\right)=\frac{h^{2}}{3}\left[V_{l+1 / 2}^{j}+V_{l}^{j}+V_{l-1 / 2}^{j}+h^{2}\left(\frac{1}{4}+b\right) V_{20}\right]+\hat{T}_{1 l}^{j}, \\
& {\left[A_{00}+\frac{h^{2}}{12}\left(A_{20}-\frac{2\left(A_{10}\right)^{2}}{A_{00}}\right)\right]\left[V_{l+1}^{j}-2 V_{l}^{j}+V_{l-1}^{j}\right]} \\
& \quad+\frac{h^{2}}{12}\left[10 U_{t l}^{j}+\left(1-\frac{h A_{10}}{A_{00}}\right) U_{t l+1}^{j}+\left(1+\frac{h A_{10}}{A_{00}}\right) U_{t l-1}^{j}+6 k U_{02}\right] \\
& =\frac{h^{2}}{3}\left[\left(1-\frac{h A_{10}}{2 A_{00}}\right) F_{l+1 / 2}^{j}+\left(1+\frac{h A_{10}}{2 A_{00}}\right) F_{l-1 / 2}^{j}+F_{l}^{j}+h^{2}\left(a+\frac{1}{4}\right) U_{20} \alpha_{l}^{(1) j}\right. \\
& \left.\quad+h^{2}\left(b+\frac{1}{4}\right) V_{20} \alpha_{l}^{(2) j}\right]+\hat{T}_{2 l}^{j} .
\end{aligned}
$$

Comparing (4.59)-(4.60) with the consistency conditions (4.9)-(4.10), we obtain

$$
\begin{aligned}
\hat{T}_{1 l}^{j}= & \frac{-h^{4}}{3}\left(b+\frac{1}{4}\right) V_{20}+O\left(k^{2} h^{2}+k h^{4}+h^{6}\right), \\
\hat{T}_{2 l}^{j}= & k h^{2}\left(\frac{1}{2}-\theta\right) U_{02}-\frac{h^{4}}{3}\left[\left(a+\frac{1}{4}\right) U_{20} \alpha_{l}^{(1) j}+\left(b+\frac{1}{4}\right) V_{20} \alpha_{l}^{(2) j}\right] \\
& +O\left(k^{2} h^{2}+k h^{4}+h^{6}\right) .
\end{aligned}
$$

The proposed method (3.39)-(3.40) to be of $O\left(k^{2}+k h^{2}+h^{4}\right)$, the coefficients of $k h^{2}$ and $h^{4}$ in (4.61)-(4.62) must be zero.

Thus

$$
\theta=\frac{1}{2}, \quad a=b=\frac{-1}{4} .
$$

Whenever $A=A(x, t, u)$, the differential equation (1.1) becomes quasi-linear. In order to solve the quasi-linear differential equation when $A=A(x, t, u)$, we need to modify the method (3.39)-(3.40). The details of the technique to write numerical schemes for quasilinear problems are discussed in $[18,23,26,28]$. The same technique can be used here to write a numerical scheme for quasi-linear differential equation (1.1) when $A=A(x, t, u)$.

\section{Stability analysis}

For stability analysis of the proposed method, we consider the linearized KS equation without second order derivative term

$$
u_{t}+\gamma u_{x}+\delta u_{x x x x}=0
$$


where $\gamma, \delta>0$ are constants. Applying the proposed method (3.39)-(3.40) to the linearized KS equation (5.1) and neglecting local truncation errors, we obtain the following nonpolynomial schemes in a coupled form:

$$
\begin{aligned}
12\left(u_{l-1}^{j+1}-2 u_{l}^{j+1}+u_{l+1}^{j+1}\right)-h^{2}\left(v_{l-1}^{j+1}+10 v_{l}^{j+1}+v_{l+1}^{j+1}\right) & \\
= & -12\left(u_{l-1}^{j}-2 u_{l}^{j}+u_{l+1}^{j}\right)+h^{2}\left(v_{l-1}^{j}+10 v_{l}^{j}+v_{l+1}^{j}\right), \\
(1- & 3 \gamma \lambda h) u_{l-1}^{j+1}+10 u_{l}^{j+1}+(1+3 \gamma \lambda h) u_{l+1}^{j+1} \\
& +\lambda\left[\left(6 \delta+\frac{\gamma \alpha h^{3}}{2}\right) v_{l-1}^{j+1}-12 \delta v_{l}^{j+1}+\left(6 \delta+\frac{\gamma \alpha h^{3}}{2}\right) v_{l+1}^{j+1}\right] \\
= & (1+3 \gamma \lambda h) u_{l-1}^{j}+10 u_{l}^{j}+(1-3 \gamma \lambda h) u_{l+1}^{j} \\
& -\lambda\left[\left(6 \delta+\frac{\gamma \alpha h^{3}}{2}\right) v_{l-1}^{j}-12 \delta v_{l}^{j}+\left(6 \delta-\frac{\gamma \alpha h^{3}}{2}\right) v_{l+1}^{j}\right] .
\end{aligned}
$$

To apply the von Neumann linear stability method, we consider that the numerical solutions are given by $u_{l}^{j}=\rho^{j} e^{i \eta l}$ and $v_{l}^{j}=\sigma^{j} e^{i \eta l}$ for $i=\sqrt{-1}$, where $\rho, \sigma$ are amplification factors and $\eta$ is the phase angle. Substituting these in Schemes (5.2)-(5.3), we obtain the following matrix form:

$$
\boldsymbol{A} \boldsymbol{y}^{j+1}=\boldsymbol{B y}^{j}
$$

where

$$
\begin{aligned}
& \boldsymbol{y}=\left[\begin{array}{l}
\boldsymbol{u} \\
\boldsymbol{v}
\end{array}\right], \quad \boldsymbol{A}=\left[\begin{array}{ll}
p & q \\
r & s
\end{array}\right], \quad \boldsymbol{B}=\left[\begin{array}{ll}
p & q \\
r^{*} & s
\end{array}\right], \\
& p=-\sin ^{2} \frac{\eta}{2}, \quad q=-\frac{h^{2}}{4}\left(1-\frac{1}{3} \sin ^{2} \frac{\eta}{2}\right), \\
& r=\left(1-\frac{1}{3} \sin ^{2} \frac{\eta}{2}\right)+i \frac{\gamma \lambda h}{8} \sin \eta, \quad r^{*}=\left(1-\frac{1}{3} \sin ^{2} \frac{\eta}{2}\right)-i \frac{\gamma \lambda h}{8} \sin \eta, \\
& d=-\frac{\delta \lambda}{2} \sin ^{2} \frac{\eta}{2}-\frac{i \gamma \alpha \lambda h^{3}}{48} \sin \eta .
\end{aligned}
$$

Now, define the amplification matrix $\boldsymbol{H}$ as

$$
\boldsymbol{H}=\boldsymbol{A}^{-1} \boldsymbol{B} .
$$

Let the eigenvalues of the amplification matrix $\boldsymbol{H}$ be $\omega=\omega_{1}, \omega_{2}$. The von Neumann necessary condition for linear stability of system (5.2)-(5.3) is that $\max |\omega| \leq 1$. By direct calculation, the eigenvalues of $\boldsymbol{H}$ are given by

$$
\omega_{1}=-1, \quad \omega_{2}=\frac{C+i D}{C^{*}-i D},
$$

where

$$
C=-\frac{h^{2}}{4}\left(1-\frac{1}{3} \sin ^{2} \frac{\eta}{2}\right)^{2}+\frac{\delta \lambda}{2} \sin ^{4} \frac{\eta}{2},
$$




$$
\begin{aligned}
& C^{*}=-\frac{h^{2}}{4}\left(1-\frac{1}{3} \sin ^{2} \frac{\eta}{2}\right)^{2}-\frac{\delta \lambda}{2} \sin ^{4} \frac{\eta}{2} \\
& D=\frac{\gamma \lambda h}{8}\left(1-\frac{1}{3} \sin ^{2} \frac{\eta}{2}\right)+\frac{\gamma \alpha \lambda h^{3}}{48} \sin ^{2} \frac{\eta}{2} \sin \eta .
\end{aligned}
$$

It is noticed that $\max \left|\omega_{1}\right|=1$ and $\max \left|\omega_{2}\right| \leq 1$ if

$$
\frac{\delta \lambda}{2} h^{2}\left(1-\frac{1}{3} \sin ^{2} \frac{\eta}{2}\right)^{2} \sin ^{4} \frac{\eta}{2} \geq 0
$$

Inequality (5.6) is true for all values of phase angle $\eta$. Hence system (5.3)-(5.4) is unconditionally stable.

\section{Numerical results}

In this section, we apply the proposed spline in compression method to the GKS equation, KS equation, and EFK equation with different parameters. The exact solutions are provided as a test procedure. The initial and boundary conditions can be obtained from the exact solutions. Whenever PDE (1.1) is quasi-linear or nonlinear, the proposed method builds a coupled nonlinear block system. In the following examples, we use Newton's block Gauss-Seidel iteration method [30-33] for the solution of a coupled nonlinear block system. In each case iterations were stopped when the absolute error tolerance $\leq 10^{-10}$ is achieved.

Example 1 (Kuramoto-Sivashinsky equation)

$$
u_{t}+u u_{x}+u_{x x}+u_{x x x x}=0, \quad-30<x<30, t>0 .
$$

The exact solution $[13,34]$ is given by

$$
u(x, t)=\beta_{0}+\frac{15}{19} \sqrt{\frac{11}{19}}\left\{-9 \tanh \left(\kappa\left(x-\beta_{0} t-x_{0}\right)\right)+11 \tanh ^{3}\left(\kappa\left(x-\beta_{0} t-x_{0}\right)\right)\right\}
$$

For computation, we choose the values $\beta_{0}=5, \kappa=\frac{1}{2} \sqrt{\frac{11}{19}}, x_{0}=-12$ in the solution domain [-30,30] with $h=1 / 150$ and $k=1 / 100$. To check the accuracy of the proposed method, we compute the global relative errors (GRE) defined using the formula

$$
\mathrm{GRE}=\frac{\sum_{l=1}^{L}\left|u_{l}^{j}-U_{l}^{j}\right|}{\sum_{l=1}^{L}\left|U_{l}^{j}\right|}
$$

where $u_{l}^{j}$ and $U_{l}^{j}$ denote the numerical and exact solution values at the grid point $\left(x_{l}, t_{j}\right)$, respectively. We compare our numerical results with the results given in $[13,15,18]$. The GREs for the solutions of (6.1) are presented in Table 1a at different time levels. We show the comparison of exact and numerical solutions at various time levels in Fig. 1. In Table $1 \mathrm{~b}$, the GRE is compared with the results given in [15] to exhibit the effect of change in the number of grid points.

Example 2 (Kuramoto-Sivashinsky equation)

$$
u_{t}+u u_{x}-u_{x x}+u_{x x x x}=0, \quad-50<x<50, t>0 .
$$


Table 1 Example 1: The global relative errors

\begin{tabular}{lllll} 
(a) & & & \\
\hline$t$ & $\begin{array}{l}\text { Proposed method } \\
(3.39)-(3.40)\end{array}$ & $\begin{array}{l}\text { Method discussed } \\
\text { in [18] }\end{array}$ & $\begin{array}{l}\text { Method discussed } \\
\text { in [15] }\end{array}$ & $\begin{array}{l}\text { Method discussed } \\
\text { in [13] }\end{array}$ \\
\hline 1 & $1.9827(-05)$ & $6.0297(-05)$ & $3.8173(-04)$ & $6.7923(-04)$ \\
2 & $3.1204(-05)$ & $9.9303(-05)$ & $5.5114(-04)$ & $1.1503(-03)$ \\
3 & $8.2856(-05)$ & $1.3064(-04)$ & $7.0398(-04)$ & $1.5941(-03)$ \\
4 & $8.5826(-05)$ & $1.6060(-04)$ & $8.6366(-04)$ & $2.0075(-03)$ \\
\hline
\end{tabular}

\begin{tabular}{|c|c|c|c|c|c|c|}
\hline \multirow[t]{2}{*}{$t$} & \multicolumn{2}{|l|}{$L+1=200$} & \multicolumn{2}{|l|}{$L+1=300$} & \multicolumn{2}{|l|}{$L+1=400$} \\
\hline & $\begin{array}{l}\text { Proposed } \\
\text { method } \\
(3.39)-(3.40)\end{array}$ & $\begin{array}{l}\text { Method } \\
\text { discussed } \\
\text { in [15] }\end{array}$ & $\begin{array}{l}\text { Proposed } \\
\text { method } \\
(3.39)-(3.40)\end{array}$ & $\begin{array}{l}\text { Method } \\
\text { discussed } \\
\text { in [15] }\end{array}$ & $\begin{array}{l}\text { Proposed } \\
\text { method } \\
(3.39)-(3.40)\end{array}$ & $\begin{array}{l}\text { Method } \\
\text { discussed } \\
\text { in [15] }\end{array}$ \\
\hline 1 & $3.4024(-05)$ & $2.1335(-04)$ & $2.5260(-05)$ & $1.2335(-04)$ & $2.3821(-05)$ & $6.6956(-05)$ \\
\hline 2 & $5.8546(-05)$ & $3.0874(-04)$ & $4.6256(-05)$ & $1.6780(-04)$ & $2.6792(-05)$ & $9.6417(-05)$ \\
\hline 3 & $8.0178(-05)$ & $3.9500(-04)$ & $6.5287(-05)$ & $2.0791(-04)$ & $4.5023(-05)$ & $1.0947(-04)$ \\
\hline 4 & $9.8195(-05)$ & $4.8479(-04)$ & $8.2460(-05)$ & $2.5018(-04)$ & $8.0236(-05)$ & $1.2600(-04)$ \\
\hline
\end{tabular}

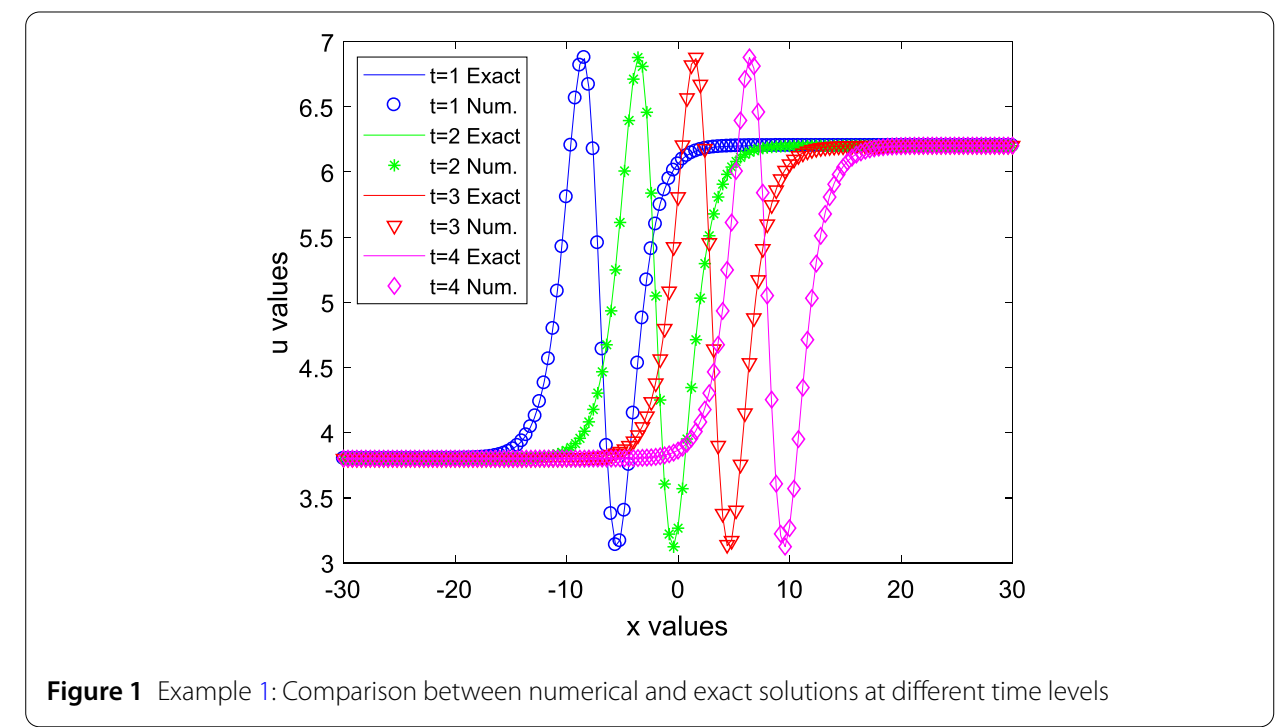

The exact solution $[13,34]$ is given by

$$
u(x, t)=\beta_{0}+\frac{15}{19 \sqrt{19}}\left\{-3 \tanh \left(\kappa\left(x-\beta_{0} t-x_{0}\right)\right)+\tanh ^{3}\left(\kappa\left(x-\beta_{0} t-x_{0}\right)\right)\right\}
$$

For computation, we choose the values $\beta_{0}=5, \kappa=\frac{1}{2 \sqrt{19}}, x_{0}=-25$ in the interval $[-50,50]$ with $h=1 / 200$ and $k=1 / 100$. In Table 2 , the GRE is compared with the results given in [13, 15]. At various time levels, we present the graph between numerical and exact solutions in Fig. 2.

Example 3 (Generalized Kuramoto-Sivashinsky equation)

$$
u_{t}+u u_{x}+u_{x x}+4 u_{x x x}+u_{x x x x}=0, \quad-30<x<30, t>0 .
$$


Table 2 Example 2: The global relative errors

\begin{tabular}{rlll}
\hline$t$ & Proposed method (3.39)-(3.40) & Method discussed in [15] & Method discussed in [13] \\
\hline 6 & $3.9866(-07)$ & $6.5093(-06)$ & $7.8808(-06)$ \\
8 & $4.7197(-07)$ & $7.1315(-06)$ & $9.5324(-06)$ \\
10 & $5.2943(-07)$ & $7.3103(-06)$ & $1.0891(-06)$ \\
12 & $5.8154(-07)$ & $8.7766(-06)$ & $1.1793(-06)$ \\
\hline
\end{tabular}

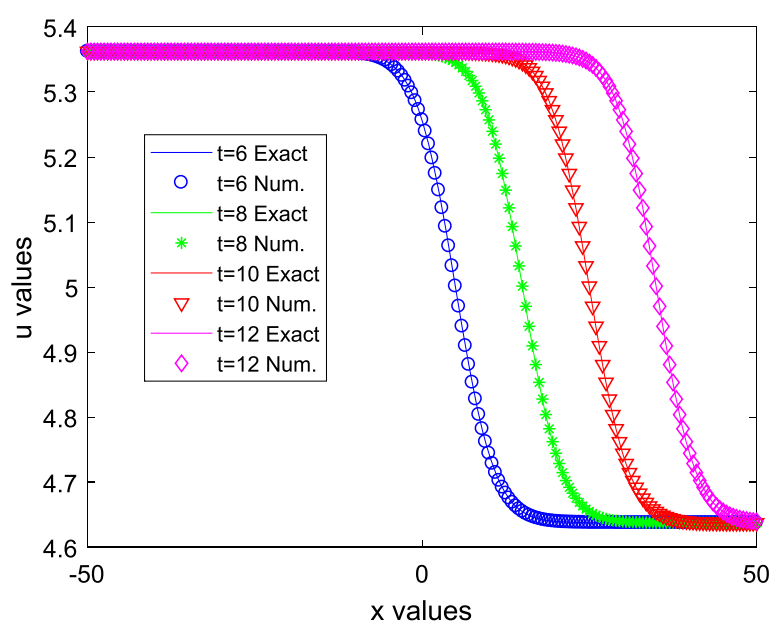

Figure 2 Example 2: Comparison between numerical and exact solutions at different time levels

Table 3 Example 3: The global relative errors

\begin{tabular}{llll}
\hline$t$ & Proposed method (3.39)-(3.40) & Method discussed in [18] & Method discussed in [13] \\
\hline 1 & $1.1734(-05)$ & $8.4059(-05)$ & $2.5945(-02)$ \\
2 & $3.9568(-05)$ & $2.7154(-04)$ & $2.7959(-02)$ \\
3 & $7.8924(-05)$ & $5.3351(-04)$ & $2.6701(-02)$ \\
4 & $1.2925(-04)$ & $8.5210(-04)$ & $3.5172(-02)$ \\
\hline
\end{tabular}

The exact solution $[13,34]$ is given by

$$
\begin{aligned}
u(x, t)= & \beta_{0}+9-15\left\{\tanh \left(\kappa\left(x-\beta_{0} t-x_{0}\right)\right)+\tanh ^{2}\left(\kappa\left(x-\beta_{0} t-x_{0}\right)\right)\right. \\
& \left.-\tanh ^{3}\left(\kappa\left(x-\beta_{0} t-x_{0}\right)\right)\right\} .
\end{aligned}
$$

For computation, we choose the values $\beta_{0}=6, \kappa=0.5, x_{0}=-10$ in the solution domain [-30,30] with $h=1 / 10$ and $k=1 / 10,000$. The GRE is reported in Table 3 and the results are compared with the results given in $[13,18]$. In Fig. 3, we present the comparison of exact and numerical solutions at $t=1$.

Example 4 (Extended Fisher-Kolmogorov equation)

$$
u_{t}-u_{x x}+\gamma u_{x x x x}+u^{3}-u=0, \quad-4<x<4, t>0 .
$$




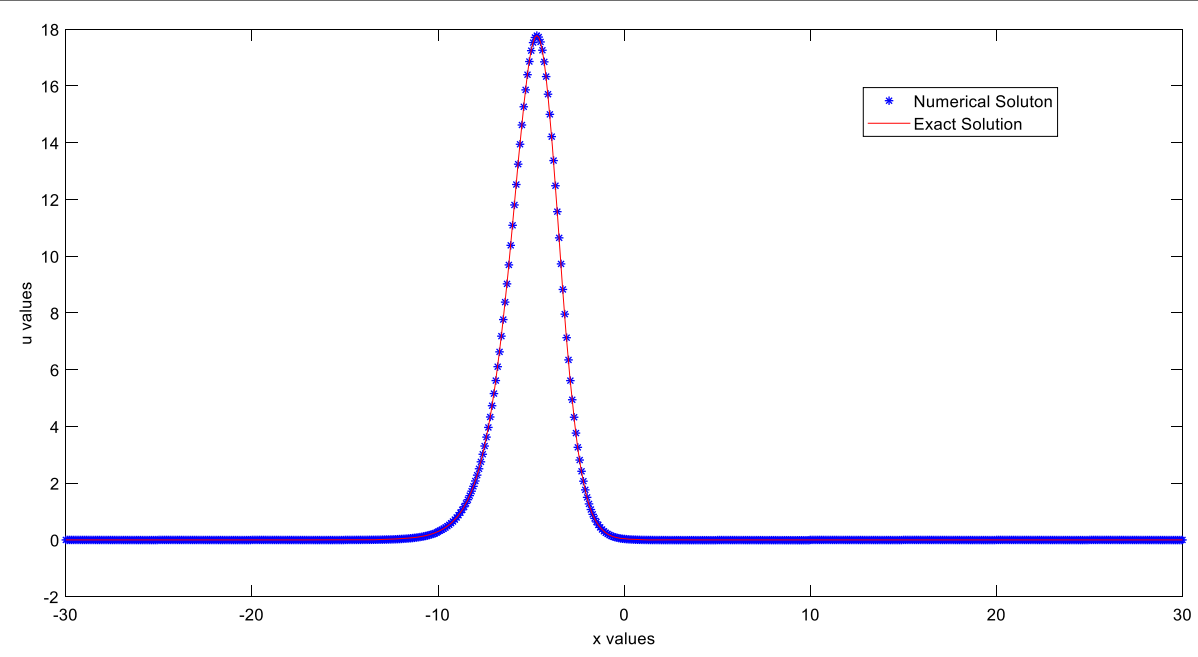

Figure 3 Example 3: Comparison between numerical and exact solutions at $t=1$

The initial and boundary conditions [20] are given by

$$
\begin{aligned}
& u(x, 0)=-\sin (\pi x), \quad-4 \leq x \leq 4, \\
& u(-4, t)=0, \quad u(4, t)=0, \quad t>0, \\
& u_{x x}(-4, t)=0, \quad u_{x x}(4, t)=0 .
\end{aligned}
$$

For the above conditions, we plotted graphs (Fig. 4a-4c) of the computed solution at different time levels. We observe that the behavior of the numerical solution at $\gamma=0$ and $\gamma=0.0001$ is almost similar. However, we notice that as time $t$ increases, the solution curves fall to zero rapidly for $\gamma=0.1$, which ensures the stabilizing character of the EFK equation.

\section{Final discussion}

In this paper, we have discussed a new two-level implicit numerical method in a coupled form based on spline in compression approximations for the solution of time-dependent quasi-linear biharmonic equations. The proposed spline method uses only three spatial grid points $x_{l}, x_{l \pm 1 / 2}$, and no fictitious points for incorporating the boundary conditions are needed. The numerical solution of $u_{x x}$ is obtained as a by-product of the method. The numerical results clearly suggest that the scheme produces better results in comparison with the existing results given in $[13,15,18]$. It is noted from Tables 1,2 and 3 that the accuracy of the solution for the KS equation decreases with time due to the time truncation errors of the time derivative term. The graphical illustration of numerical solution at various time levels establishes similar features as those existing in the literature. The proposed method is easily implemented and approximates the exact solution of highly nonlinear GKS equation and KS equation. The graphical results of EFK equation obtained by the proposed scheme exhibit the correct physical behavior for different values of $\gamma$. Similar patterns have been depicted in [20]. It is expected that the proposed method will be helpful in solving other nonlinear 1D time-dependent biharmonic problems in applied sciences and engineering. 


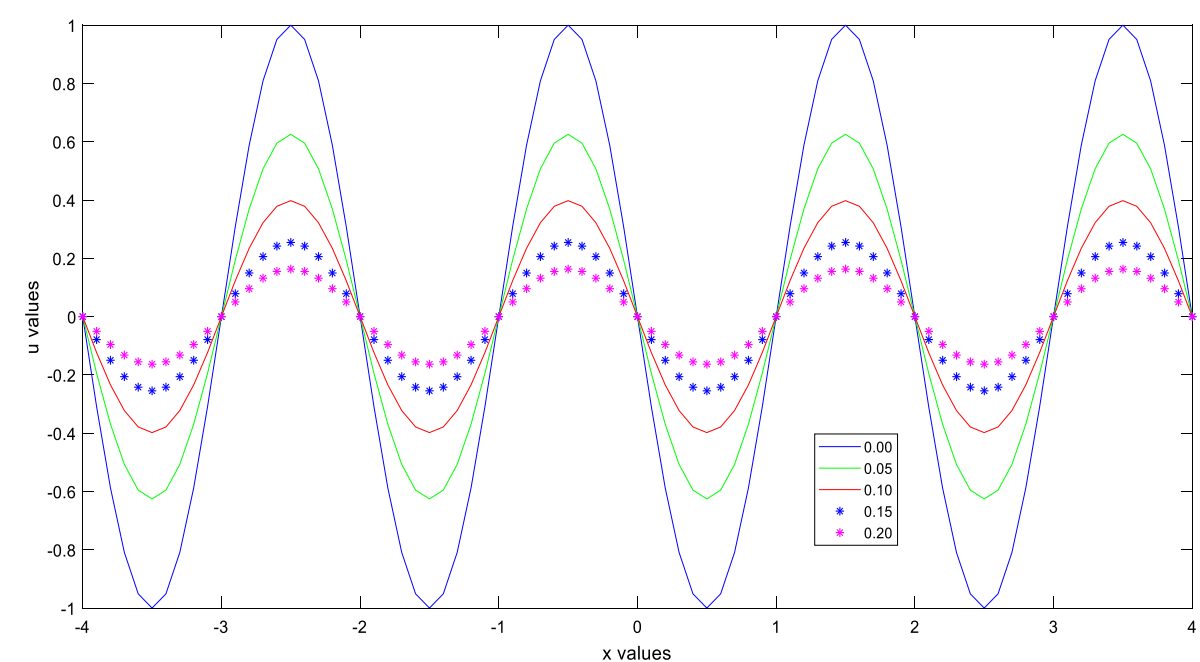

(a)

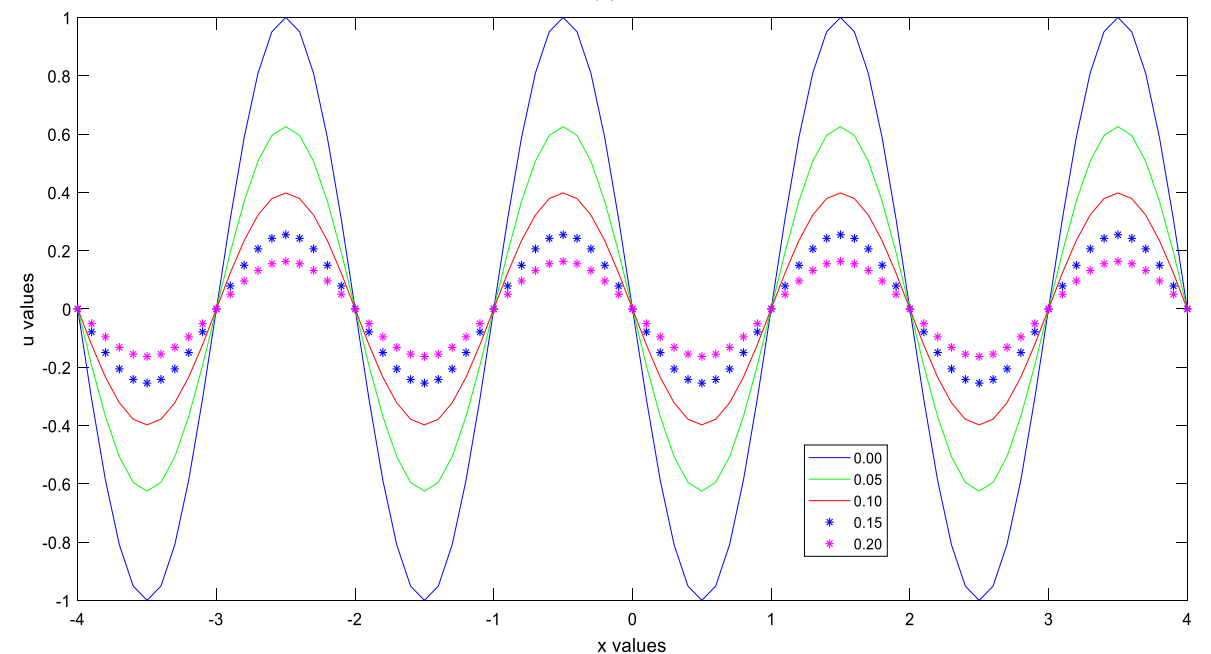

(b)

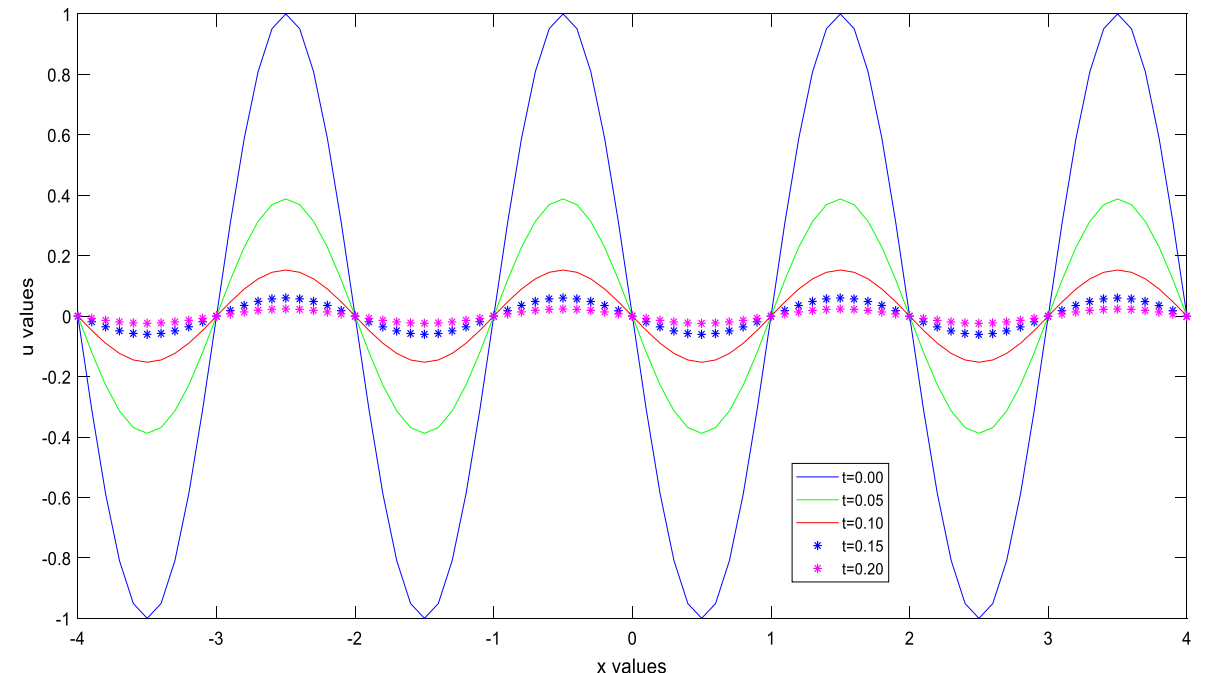

(c)

Figure 4 Example 4: (a) The profiles of $u(x, t)$ versus $x$ for $\gamma=0$. (b) The profiles of $u(x, t)$ versus $x$ for $\gamma=0.0001$. (c) The profiles of $u(x, t)$ versus $x$ for $\gamma=0.1$ 
Acknowledgements

The authors thank the reviewers for their valuable suggestions, which substantially improved the standard of the paper.

\section{Funding}

This research work is supported by CSIR-SRF, Grant No: 09/045(1161)/2012-EMR-I.

\section{Competing interests}

The authors declare that they have no competing interests.

Authors' contributions

All authors drafted the manuscript, and they read and approved the final version.

\section{Author details}

'Department of Applied Mathematics, South Asian University, New Delhi, India. ${ }^{2}$ Department of Mathematics, Faculty of Mathematical Sciences, University of Delhi, Delhi, India. ${ }^{3}$ Department of Mathematics, Sri Venkateswara College, University of Delhi, New Delhi, India.

\section{Publisher's Note}

Springer Nature remains neutral with regard to jurisdictional claims in published maps and institutional affiliations.

\section{Received: 3 August 2018 Accepted: 9 October 2018 Published online: 20 October 2018}

\section{References}

1. Aronson, D.G., Weinberger, H.F.: Multidimensional nonlinear diffusion arising in population genetics. Adv. Math. 30, 33-67 (1978)

2. Conte, R.: Exact solutions of nonlinear partial differential equations by singularity analysis. In: Lecture Notes in Physics, pp. 1-83. Springer, Berlin (2003)

3. Dee, G.T., van Saarloos, W.: Bistable systems with propagating fronts leading to pattern formation. Phys. Rev. Lett. 60 , 2641-2644 (1988)

4. Hooper, A.P., Grimshaw, R.: Nonlinear instability at the interface between two viscous fluids. Phys. Fluids 28, 37-45 (1985)

5. Hornreich, R.M., Luban, M., Shtrikman, S.: Critical behaviour at the onset of k-space instability at the $\lambda$ line. Phys. Rev Lett. 35, 1678-1681 (1975)

6. Kuramoto, Y., Tsuzuki, T.: Persistent propagation of concentration waves in dissipative media far from thermal equilibrium. Prog. Theor. Phys. 55, 356-369 (1976)

7. Saprykin, S., Demekhin, E.A., Kalliadasis, S.: Two-dimensional wave dynamics in thin films. I. Stationary solitary pulses. Phys. Fluids 17, 117105 (2005)

8. Sivashinsky, G.l.: Instabilities, pattern-formation, and turbulence in flames. Annu. Rev. Fluid Mech. 15, 179-199 (1983)

9. Tatsumi, T.: Irregularity, regularity and singularity of turbulence. In: Turbulence and Chaotic Phenomena in Fluids. lutam, pp. 1-10 (1984)

10. Zhu, G.: Experiments on director waves in nematic liquid crystals. Phys. Rev. Lett. 49, 1332-1335 (1982)

11. Xu, Y., Shu, C.W.: Local discontinuous Galerkin methods for the Kuramoto-Sivashinsky equations and the Ito-type coupled KdV equations. Comput. Methods Appl. Mech. Eng. 195, 3430-3447 (2006)

12. Khater, A.H., Temsah, R.S.: Numerical solutions of the generalized Kuramoto-Sivashinsky equation by Chebyshev spectral collocation methods. Comput. Math. Appl. 56, 1456-1472 (2008)

13. Lai, H., Ma, C.: Lattice Boltzmann method for the generalized Kuramoto-Sivashinsky equation. Physica A 388 1405-1412 (2009)

14. Uddin, M., Haq, S., Siraj-ul-Islam: A mesh-free numerical method for solution of the family of Kuramoto-Sivashinsky equations. Appl. Math. Comput. 212, 458-469 (2009)

15. Mittal, R.C., Arora, G.: Quintic B-spline collocation method for numerical solution of the Kuramoto-Sivashinsky equation. Commun. Nonlinear Sci. Numer. Simul. 15, 2798-2808 (2010)

16. Lakestani, M., Dehghan, M.: Numerical solutions of the generalized Kuramoto-Sivashinsky equation using B-spline functions. Appl. Math. Model. 36, 605-617 (2012)

17. Ganaiea, I.A., Arora, S., Kukreja, V.K.: Cubic Hermite collocation solution of Kuramoto-Sivashinsky equation. Int. J. Comput. Math. 93, 223-235 (2016)

18. Mohanty, R.K., Kaur, D.: Numerov type variable mesh approximations for 1D unsteady quasi-linear biharmonic problem: application to Kuramoto-Sivashinsky equation. Numer. Algorithms 74, 427-459 (2017)

19. Rashidinia, J., Jokar, M.: Polynomial scaling functions for numerical solution of generalized Kuramoto-Sivashinsky equation. Appl. Anal. 96, 293-306 (2017)

20. Danumjaya, P., Pani, A.K.: Orthogonal cubic spline collocation method for the extended Fisher-Kolmogorov equation J. Comput. Appl. Math. 174, 101-117 (2005)

21. Doss, L.J.T., Nandini, A.P.: An $H^{1}$-Galerkin mixed finite element method for the extended Fisher-Kolmogorov equation Int. J. Numer. Anal. Model. Ser. B 3, 460-485 (2012)

22. Stephenson, J.W.: Single cell discretizations of order two and four for biharmonic problems. J. Comput. Phys. 55, 65-80 (1984)

23. Mohanty, R.K.: An accurate three spatial grid-point discretization of $O\left(k^{2}+h^{4}\right)$ for the numerical solution of one-space dimensional unsteady quasi-linear biharmonic problem of second kind. Appl. Math. Comput. 140, 1-14 (2003)

24. Jain, M.K., Iyenger, S.R.K., Pillai, A.C.R.: Difference schemes based on spline in compression for the solution of conservative laws. Comput. Methods Appl. Mech. Eng. 38, 137-151 (1983)

25. Kadalbajoo, M.K., Patidar, K.C.: Variable mesh spline in compression for the numerical solution of singular perturbation problems. Int. J. Comput. Math. 80, 83-93 (2003) 
26. Mohanty, R.K., Gopal, V.: High accuracy non-polynomial spline in compression method for one-space dimensional quasilinear hyperbolic equations with significant first order space derivative term. Appl. Math. Comput. 238, 250-265 (2014)

27. Talwar, J., Mohanty, R.K., Singh, S.: A new spline in compression approximation for one space dimensional quasilinear parabolic equations on a variable mesh. Appl. Math. Comput. 260, 82-96 (2015)

28. Mohanty, R.K., Sharma, S.: High accuracy quasi-variable mesh method for the system of 1D quasi-linear parabolic partial differential equations based on off-step spline in compression approximations. Adv. Differ. Equ. 2017, 212 (2017)

29. Mohanty, R.K., Sharma, S.: A new two-level implicit scheme for the system of 1D quasi-linear parabolic partial differential equations using spline in compression approximations. Differ. Equ. Dyn. Syst. (2018). https://doi.org/10.1007/s12591-018-0427-5

30. Jain, M.K., Jain, R.K., Mohanty, R.K.: Fourth order difference method for the one-dimensional general quasi-linear parabolic partial differential equation. Numer. Methods Partial Differ. Equ. 6, 311-319 (1990)

31. Jain, M.K., Jain, R.K., Mohanty, R.K.: High order difference methods for system of 1D non-linear parabolic partial differential equations. Int. J. Comput. Math. 37, 105-112 (1990)

32. Mohanty, R.K., Jha, N., Evans, D.J.: Spline in compression method for the numerical solution of singularly perturbed two point singular boundary value problems. Int. J. Comput. Math. 81, 615-627 (2004)

33. Mohanty, R.K., Jha, N.: A class of variable mesh spline in compression methods for singularly perturbed two point singular boundary value problems. Appl. Math. Comput. 168, 704-716 (2005)

34. Fan, E.: Extended tanh-function method and its applications to nonlinear equations. Phys. Lett. A 277, 212-218 (2000)

\section{Submit your manuscript to a SpringerOpen ${ }^{\circ}$ journal and benefit from:}

- Convenient online submission

- Rigorous peer review

Open access: articles freely available online

- High visibility within the field

Retaining the copyright to your article

Submit your next manuscript at $>$ springeropen.com 